\title{
Factores asociados a la actitud hacia la investigación en estudiantes universitarios de enfermería
}

\author{
Factors associated with the attitude toward research in university nursing \\ students \\ Pamela Chara-Saavedra \\ Adeli Olortegui-Luna ${ }^{l}$
}

\begin{abstract}
Resumen
Objetivo: determinar qué factores se asocian con la actitud de los universitarios de enfermería hacia la investigación de una universidad de Lima Norte. Materiales y métodos: estudio descriptivo correlacional de corte transversal con enfoque cuantitativo. En el análisis descriptivo de las variables categóricas se emplearon frecuencias y porcentajes. Para el análisis bivariado se empleó la prueba de Chi-cuadrado. Para la asociación entre la variable numérica vs categórica politómica se utilizó la prueba de Kruskal-Wallis previamente de la prueba de normalidad de Shapiro-wilk con una significancia menor o igual a 0.05. Se empleó el instrumento titulado Escala de Actitudes hacia la Investigación que constaba de 30 ítems. Resultados: se muestra que la actitud hacia la investigación en los estudiantes de enfermería resultó medianamente favorable (58\%). Por otro lado, existe relación significativa entre el tiempo de trabajo ( $\mathrm{p}=0.02)$, leer artículos o libros a la semana $(\mathrm{p}=0.04)$ y la capacitación sobre investigación $(\mathrm{p}=0.01)$ con la actitud hacia la investigación. Es decir trabajar más de 10 horas (63.6\%), leer menos libros y artículos a la semana (0.8 \pm 0.6$)$ y no asistir en capacitación sobre investigación $(25.0 \%)$ se asociaron con una actitud desfavorable hacia la investigación. Conclusión: los estudiantes que trabajan más tiempo, leen menos libros y artículos a la semana y no asisten a una capacitación sobre investigación tienen una actitud desfavorable hacia la investigación. Se recomienda que las autoridades y docentes de las universidades implementen estrategias educativas con el fin de apoyar a los estudiantes con estas características sociodemográficas y fomentar la investigación a través de cursos curriculares, eventos científicos, etc.
\end{abstract}

Palabras clave: Investigación; Actitud; Estudiantes de Enfermería (Fuente: DeCS).

\begin{abstract}
Objective: to determine what factors are associated with the attitude of university students of nursing towards the research of a university in Lima Norte. Materials and methods: cross-sectional descriptive correlational study with a quantitative approach. In the descriptive analysis of the categorical variables frequencies and percentages were used. For the bivariate analysis, the Chi-square test was used. For the association between the numerical variable vs the categorical polytomics, the Kruskal-Wallis test was previously used for the normality test of Shapiro-wilk with a significance less than or equal to 0.05. The instrument entitled Scale of Attitudes toward Research was used, which consisted of 30 items. Results: it is shown that the attitude toward research in nursing students was moderately favorable (58\%). On the other hand, there is a significant relationship between working time $(\mathrm{p}=0.02)$, reading articles or books per week $(\mathrm{p}=0.04)$ and research training $(\mathrm{p}=0.01)$ with attitude toward research. That is, working more than 10 hours $(63.6 \%)$, reading fewer books and articles per week $(0.8 \pm 0.6)$ and not attending research training $(25.0 \%)$ were associated with an unfavorable attitude towards research. Conclusions: students who work longer, read fewer books and articles per week and do not attend research training have an unfavorable attitude towards research. It is recommended that the authorities and teachers of the universities implement educational strategies in order to support students with these sociodemographic characteristics and encourage research through curricular courses, scientific events, etc.
\end{abstract}

Key words: Research; Attitude; Nursing students (Source: DeCS).

Para citar:

Chara P, Olortegui A. Factores asociados a la actitud hacia la investigación en estudiantes universitarios de enfermería. CASUS. 2018;3(2):83-88.

${ }^{1}$ Universidad Católica Sedes Sapientiae. Estudiante de Enfermería. Correo electrónico: charasaavedra.pamelayessica@gmail.com
Fecha de recepción: 25-05-18

Fecha de envío a pares: 26-05-18

Fecha de aprobación por pares: 22-08-18

Fecha de aceptación: 26-08-18 


\section{INTRODUCCIÓN}

El escaso número de investigaciones científicas realizadas por estudiantes en todas las universidades, es el síntoma que condensa lo mucho que se tiene que mejorar en el sistema educativo peruano (1). Las raíces de este problema son muy profundas, pues se inician desde los inferiores niveles de educación. Es cuando los docentes no motivan a los estudiantes sobre una correcta lectura y análisis crítico sobre la búsqueda de la información $(1,2)$. Este problema se agrava cuando una universidad no promueve la investigación en su comunidad y por consecuencia no formaría profesionales con actitudes creativas y de desarrollo $(1,2)$.

En diversas universidades del Perú, la investigación científica para los estudiantes no es una actividad curricular ya que no hay una correcta dedicación para su desarrollo. Por ello, existe un escaso número de estudiantes con capacidad de investigar. La actividad investigativa pasa a ser un trabajo de recibir informaciones y cumplir una tarea hasta llegar al punto que su comunidad universitaria, por ello se convierte en un ambiente hostil en la que se baza enseñanzas tradicionales $(3,4)$. Sin embargo, si las universidades consideran a la investigación dentro de un curso, permitiría al estudiante adquirir ciertas capacidades y competencias investigativas obteniendo como productos: tesis, artículos científicos, ensayos y con ello puede tener la posibilidad de presentarse a congresos científicos en ciencias de salud $(5,6)$.

Sin embargo, el principal límite para favorecer la investigación en los universitarios es el "curso de actualización académica" que ampara la Superintendencia Nacional de Educación Superior Universitaria (SUNEDU) (7). Este curso es una de las modalidades para obtener el grado de bachiller y/o título profesional para licenciatura de una manera fácil y rápida. Esto disminuye o demerita el valor a los procesos de investigación que permiten al estudiante a obtener un grado o título por medio de la realización de la tesis (7-9).

En el 2015 se realizó un informe Bienal sobre la realidad universitaria en el Perú. En sus indicadores de documentos citables (artículos, conference papers y reviews), que la investigación científica en el Perú atraviesa una crisis, no solo por la baja producción de estos, sino también por la baja calidad (3). Además en el 2010 solo se han publicado 927 documentos citables en ese año y para el 2015 se llegaron a publicar 1610, con una tasa promedio anual del $12 \%$. A pesar de ser muy significativa a nivel nacional, la investigación en el Perú se sigue ubicando por debajo de otros países a nivel latinoamericano. En los países de Colombia y Chile actualmente desarrollan aproximadamente entre 7 mil a 10 mil documentos citables por año (3).

Por esa razón, en la actualidad, la actitud hacia la investigación es de suma importancia en todos los universitarios. A través de ella se genera un análisis frente a problemas que puedan ser solucionados de manera metodológica y reflexiva (10). La actitud hacia la investigación son predisposiciones referidas por los estudiantes, provenientes de estados psicológicos internos (creencias y cogniciones) con una carga afectiva a favor o en contra de la investigación (10). La misma está vinculada a la formación profesional de los estudiantes porque da inicio al desarrollo de capacidades de abstracción, análisis crítico y síntesis (10).

Existen ciertos factores que podrían contribuir a una actitud desfavorable o favorable hacia la investigación por parte de los estudiantes que deberían ser tomados en cuenta por las universidades. La realidad actual de los estudiantes universitarios es que paralelamente al estudio tienen otras actividades como una ocupación laboral (11). Asimismo, a los estudiantes no se les motiva o ellos no tienen el interés de buscar informaciones en artículos científicos, libros u otras fuentes confiables (12). Por último, no hay una capacitación constante para los estudiantes que pueda favorecer actitudes investigativas. Sin embargo, si los estudiantes de pregrado se involucrasen en eventos científicos, capacitaciones, cursos, talleres, jornadas o congresos científicos estarían motivando la 
investigación y publicación científica en ellos (13$15)$.

Considerando lo mencionado anteriormente no cabe duda de la importancia que tiene la actitud hacia la investigación en el pregrado. Sin embargo, se presentan ciertas limitaciones para favorecer una actitud positiva hacia la investigación por parte de los universitarios de enfermería. Razón por la cual el objetivo del estudio fue determinar qué factores se asocian con la actitud hacia la investigación en estudiantes de enfermería de una universidad privada de LimaNorte.

\section{MATERIALES Y MÉTODOS}

Se realizó un estudio descriptivo correlacional de corte transversal con enfoque cuantitativo. Se ejecutó un censo de la población de 100 estudiantes de la carrera de enfermería, de ambos sexos desde 1er ciclo hasta 10 mo ciclo de una universidad privada de Lima Norte. Teniendo en cuenta los criterios de inclusión se tomaron en cuenta solo a estudiantes de la carrera de enfermería mayores de 18 años. Se excluyeron a estudiantes de otras carreras y que no desearon participar.

La variable principal del presente estudio fue la actitud hacia la investigación. La misma se midió a través del instrumento titulado Escala de Actitudes hacia la Investigación "EACIN" que constaba de 30 ítems. La actitud hacia la investigación se categorizó en: favorable >100; medianamente favorable $\geq 89-\leq 100 ; \quad$ y desfavorable $<89$. Asimismo, esta variable se dispone en tres dimensiones: el componente afectivo (favorable $<20$; medianamente favorable $\geq 17-\leq 20$; desfavorable <17); el componente cognitivo (favorable a <56; Medianamente favorable $\geq 47$; $\leq 56$ y desfavorable <47); y el componente conductual (favorable <26; medianamente favorable $\geq 23$; $\leq 26$ y desfavorable $<23$ ) (16).

Respecto a las variables sociodemográficas se consideró la edad: variable cualitativa nominal (18-24; 25-30; 30 a más); sexo: variable cualitativa nominal (masculino y femenino); colegio de procedencia: variable cualitativa nominal (nacional, particular, parroquial); estado civil: variable cualitativa nominal (soltero, casado, divorciado, conviviente); lugar de residencia: variable cualitativa dicotómica; trabajo actual: variable cualitativa dicotómica (sí-no); condición de trabajo: variable cualitativa dicotómica (sí-no); tiempo de trabajo: variable cualitativo nominal politómica (4-6 horas diarias; 7 a 9 horas diarias; 10 a más horas diarias); horas que le dedica a la investigación: variable cualitativa nominal (30 min diarias; 30 min a 1 hora; 2-4 horas diarias); trabajo de investigación: variable cualitativa dicotómica (sí-no). Definida por la siguiente pregunta: ¿ $\mathrm{Ha}$ realizado algún trabajo de investigación como monografías, artículo científico, informes, Proceso de cuidado enfermería (PCE)?; cantidad de libros $o$ artículos que lee a la semana: variable cuantitativa; curso de capacitación: variable cualitativa dicotómica (sí-no). Definida por la siguiente pregunta: ¿ha recibido algún curso de capacitación sobre investigación este año?

Una vez recolectados los datos se utilizó el programa estadístico Stata versión 14. En el análisis descriptivo de las variables categóricas se emplearon frecuencias y porcentajes. Para el análisis bivariado se empleó la prueba de Chicuadrado con una significancia menor o igual a 0.05 . Para la asociación entre la variable numérica vs categórica politómica se utilizó la prueba de Kruskal-Wallis previamente de la prueba de normalidad de Shapiro-Wilk.

El presente estudio fue revisado y aprobado por un Comité de Ética de Investigación y no comportó riesgos a la salud de los encuestados. Para la protección de los sujetos involucrados se respetó la confidencialidad y se aplicó la política de protección de datos de los mismos.

\section{RESULTADOS}

En la figura 1 se muestra que la actitud hacia la investigación en los estudiantes de enfermería resultó medianamente favorable (58\%).

Respecto a las características sociodemográficos se muestra que de los 100 estudiantes de la carrera de 
Tabla 1. Características descriptivas de la muestra

\begin{tabular}{llll}
\hline & & n & \% \\
\hline Edad & & & \\
& $18-24$ años & 63 & 63 \\
& $25-30$ años & 23 & 23 \\
Sexo & $>30$ & 14 & 14
\end{tabular}

$\begin{array}{llll}\text { Sexo } & & & \\ & \text { Femenino } & 82 & 82 \\ & \text { Masculino } & 18 & 18\end{array}$

Colegio

\begin{tabular}{|c|c|c|c|}
\hline & Nacional & 69 & 69 \\
\hline & Particular & 22 & 22 \\
\hline & Parroquial & 9 & 9 \\
\hline \multicolumn{4}{|c|}{ Estado Civil } \\
\hline & Soltero & 90 & 90 \\
\hline & Casado & 3 & 3 \\
\hline & Divorciado & 7 & 7 \\
\hline \multicolumn{4}{|c|}{ Lugar de residencia } \\
\hline & Urbano & 94 & 94 \\
\hline & Rural & 6 & 6 \\
\hline \multicolumn{4}{|l|}{ Trabaja } \\
\hline & Sí & 54 & 54 \\
\hline & No & 46 & 46 \\
\hline \multicolumn{4}{|c|}{ Condición de trabajo } \\
\hline & Dependiente & 42 & 77,7 \\
\hline & Independiente & 12 & 22,2 \\
\hline \multicolumn{4}{|c|}{ Tiempo de trabajo } \\
\hline & 4-6 horas & 27 & 50 \\
\hline & 7-9 horas & 16 & 29,6 \\
\hline & $>10$ horas & 11 & 20,3 \\
\hline
\end{tabular}

Horas que le dedica a la investigación

$\begin{array}{lcc}\text { 30min-1 hora } & 51 & 51 \\ \text { diaria } & 42 & 42 \\ \text { 30min diarios } & 7 & 7\end{array}$

Trabajo de investigación que realizo

$\begin{array}{lcc}\text { PCE } & 43 & 43 \\ \text { Informe } & 32 & 32 \\ \text { Monografía } & 19 & 19 \\ \text { Artículo científico } & 6 & 6\end{array}$

Cantidad de libros o artículos que lee a la semana $(\mathbf{m} \pm \mathbf{D S})$ $1.08 \quad 0.81$

Curso de capacitación

\begin{tabular}{lll} 
No & 55 & 55 \\
Sí & 44 & 44 \\
\hline
\end{tabular}

Enfermería el 63\% tenían entre 18 y 24 años, y el $83 \%$ de sexo femenino. Asimismo, del total el $54 \%$ contó con un trabajo, el $77.7 \%$ trabajaba de forma dependiente y el 50\% laboraba entre 4 a 6 horas diarias. Respecto a cuántos libros o artículos leen por semana la media fue de 1.08 , es decir los estudiantes solo leen un artículo o libro a la semana. En su mayoría el trabajo de investigación que realizan los estudiantes de enfermería fue el PCE (53\%). Un 51\% de los estudiantes respondió que el tiempo que dedicaron a la investigación es de 30 minutos a 1 hora. Por último, el 55\% no recibió ningún curso de capacitación (ver tabla 1)

Tabla 2. Asociación entre el tiempo de trabajo, cantidad de libros y artículos lee a la semana, curso de capacitación y la actitud hacia la investigación

\begin{tabular}{|c|c|c|c|c|}
\hline & \multicolumn{4}{|c|}{ Atitud hacia la investigación } \\
\hline & $\begin{array}{c}\text { Favorables } \\
\%\end{array}$ & $\begin{array}{c}\text { Medianamente } \\
\%\end{array}$ & $\begin{array}{c}\text { Desfavorable } \\
\%\end{array}$ & $\mathbf{p}$ \\
\hline \multicolumn{4}{|c|}{ Tiempo de trabajo } & 0.02 \\
\hline 4-6 horas & 29.6 & 59.3 & 11.11 & \\
\hline 7-9 horas & 37.5 & 56.2 & 6.2 & \\
\hline$>10$ horas & 18.1 & 18.1 & 63.6 & \\
\hline \multicolumn{4}{|c|}{ Cantidad de libro y artículo que lee a la semana* } & 0.04 \\
\hline & $1.3 \pm 0.7$ & $1-0 \pm 0.8$ & $0.8 \pm 0.6$ & \\
\hline \multicolumn{4}{|c|}{ Capacitación } & 0.01 \\
\hline Sí & 31.8 & 63.6 & 4.5 & \\
\hline No & 21.4 & 53.5 & 25.0 & \\
\hline
\end{tabular}

En la tabla 2 se muestra que existe relación entre el tiempo de trabajo y la actitud hacia la investigación. Trabajar más de 10 horas se asocia con una actitud desfavorable (63.6\%; $\mathrm{p}=0.02)$. De la misma manera, se evidencia que la cantidad de libros o artículos que se lee a la semana se relaciona con la actitud hacia la investigación de manera desfavorable $(0.8 ; \mathrm{p}=0.01)$ evidenciando que los estudiantes no llegan a leer ni un 1 libro o artículo. De igual manera existe una relación significativa entre el curso de capacitación y la actitud hacia la investigación. No tener capacitación se asocia a tener una actitud desfavorable (25.0\%; p=0.01). El resto de asociaciones no fueron consideradas en la tabla porque no fueron estadísticamente significativas.

\section{DISCUSIÓN}

En el estudio más de la mitad de los estudiantes encuestados mostró una actitud medianamente favorable hacia la investigación. Los factores: tiempo de trabajo, la cantidad de libros o artículos que lee a la semana y participar en capacitaciones se asociaron significativamente con la actitud hacia la investigación. Sin embargo, los estudiantes que trabajan más horas; leen menos de un libro o artículos a la semana y que no recibieron ningún curso de capacitación tuvieron una actitud 
hacia la investigación desfavorable.

Trabajar más de 10 horas diarias se asocia con una actitud desfavorable hacia la investigación. Estos resultados son similares a un estudio que reportó que los estudiantes de enfermería que tienen una actividad laboral tienen un bajo rendimiento académico porque no realizan investigaciones (11). Esto es posible porque los estudiantes manifiestan que la falta de tiempo es un limitante para la realización de una investigación y esto se debe a la situación laboral y otras actividades extra académicas que presentan y que además influye en el tiempo de dedicación que tiene el estudiante hacia la investigación (17). Lamentablemente, el estudiante que tiene un trabajo dependiente, estable tiene un horario fijo, y que algunas veces está lejos de su centro de estudios.

Otro hallazgo fue que una menor cantidad de libros o artículos leídos a la semana se asocia a tener una actitud desfavorable hacia la investigación. Estos resultados fueron similares en otro estudio donde muestra que los universitarios de enfermería presentan alguna dificultad para el aprendizaje en investigación, búsqueda de información y lectura crítica (12). Destacando los elementos de búsqueda de material bibliográfico, lectura y análisis del mismo, entre otros. El proceso de adquirir estas capacidades no es fácil para los estudiantes de enfermería por no tener una lectura crítica y por ende tienen una actitud negativa hacia la investigación (12).

De igual manera, existe una relación entre la participación a cursos de capacitación y la actitud hacia la investigación. Es decir no asistir a una capacitación se asocia con tener una actitud desfavorable y por el contrario asistir a una capacitación se asocia con una actitud medianamente favorable y favorable. Es decir, que haber recibido cursos de capacitación, les motiva a investigar (13-15). Estos resultados son similares a otros estudios donde se reportó que el hecho de asistir a capacitaciones aumentó en $69 \%$ la probabilidad de tener un buen nivel de conocimientos sobre la investigación, que posibilitan el desarrollo continuo de los estudiantes en el campo científico al permitirles relacionarse con otros estudiantes y profesionales investigadores (14). En otro estudio similar también se encontró que la actitud fue buena en un $71,9 \%$, en los estudiantes que pertenecen o asisten a capacitaciones (13). Los estudiantes interesados que se agrupan podrían profundizar estos conocimientos en investigación mediante cursos, capacitaciones, talleres o jornadas y congresos científicos, ya que todas estas actividades fomentan la investigación.

Una limitación de este estudio es que los resultados obtenidos no indican causalidades entre las variables. Por otro lado, no se han abarcado factores como el tipo de familia en la cual convive el estudiante, si participa en proyectos de investigaciones, si cuenta con asesores, participa en semilleros u otros grupos de investigación. Sin embargo, los resultados ayudarían a crear estrategias para fomentar una buena actitud de los estudiantes hacia la investigación y como punto de partida de posteriores estudios en otras carreras profesionales de salud.

\section{CONCLUSIONES}

Los estudiantes de enfermería tuvieron una actitud medianamente favorable hacia la investigación. Asimismo, el tiempo de trabajo, la cantidad de libro o artículos que lee a la semana y capacitación se asociaron con la actitud hacia la investigación. Es decir, los estudiantes que trabajan más horas; leen menos de un libro o artículos a la semana y no reciben capacitación tienen una actitud desfavorable hacia la investigación.

Se recomienda que las autoridades y docentes de las universidades implementen estrategias educativas con el fin de apoyar a los estudiantes que trabajan. Para ello, se debe concientizar a la población estudiantil sobre la importancia de investigar, empezando desde los primeros ciclos de estudio para que adquiera una actitud positiva y se involucre en talleres, debates, charlas, reuniones de discusión e intercambio de informaciones. Para cambiar esta situación, es prioritario hacer cambios curriculares que garanticen la incorporación de los 
estudiantes en la investigación, desde el inicio de investigativas.

Así mismo, se recomienda realizar futuros estudios con otros factores como por ejemplo qué dificultades han presentado durante su formación académica, si cuentan con recursos para financiar su investigación, si tienen acceso a la biblioteca universitaria, laboratorios equipados, acceso a

\section{REFERENCIAS BIBLIOGRÁFICAS}

1. Falconí E. La responsabilidad del Estado y las instituciones académicas en la investigación en salud pública. Rev Peru Med Exp Salud Publica. 2007;24(4): 44041.

2. Ospina BE; Toro JA; Aristizábal AC. Rol del profesor en el proceso de enseñanza aprendizaje de la investigación en estudiantes de Enfermería de la Universidad de Antioquia, Colombia. Invest Educ Enferm. 2008;26(1): 106-114.

3. Superintendencia nacional de Educación superior universitaria (SUNEDU) "Informe Bienal sobre la realidad Universitaria Peruana," 1er edición (citado el 28 de Setiembre del 2016), a nivel nacional. Disponible en: https://drive.google.com/drive/fold ers/1SRIcEi4mFCdISG0ibKfI9Fgl e9Gn5f_5

4. Torres G. Direma, semillero de investigación en diseño y reconversión de máquinas. Scientia et technica. 2008;14(38):159-164.

5. Arroyo C. De la Cruz W, Miranda U. Dificultades para el desarrollo de investigaciones en pregrado en una universidad pública de provincia, Perú. Revista Peruana de Medicina Experimental y Salud Pública. 2008;25(4), 448-448.

6. Huamaní C, Chávez P, Domínguez W, Solano M. Producción científica estudiantil: análisis y expectativas. Rev Peru Med Exp Salud Publica. 2007; 24(4):444-

46. 60. UNAM. la carrera y desarrollar competencias internet. Para ello se debe indagar en las diferencias entre universidades públicas y privadas para verificar las problemáticas que presentan.
7. Gongora M. El Estado, la 13. Díaz C, Manrique L, Galán E, universidad peruana y el drama de la investigación científica. Escritura y Pensamiento. 2002;5(11):43-57.

8. Gutiérrez C, Mayta P. Publicación desde el pre grado en Latinoamérica: importancia, limitaciones y alternativas de solución. CIMEL. 2003; 8(1):54-

9. Ramos M, Sotomayor R. Realizar o no una tesis: razones de estudiantes de medicina de una universidad pública y factores asociados. Rev Peru Med Exp Salud $\quad$ Pública. 2008.

10. Quispe M. Actitudes de los internos de enfermería hacia la investigación y la elaboración de tesis para titularse. Perú Universidad Nacional Mayor de San Marcos 2014.

11. Trujillo J, Rosete C, Ricardez A. Principales factores que influyen en el bajo rendimiento académico en estudiantes de enfermería. Revista electrónica de investigación en enfermería FESI-

2018;7(13)

12. Ochoa K, Bello C, Villanueva M, Ruiz M, Manrique G. Percepción y actitud del universitario de enfermería sobre su formación en investigación. Rev Med Hered;
2016;27:204-215.

Apolaya M. Conocimientos, actitudes y prácticas en investigación de los estudiantes de pregrado de facultades de medicina del Perú. revista Acta Med Per. 2008;25(1)

14. Cabrera J, Cruzado C, Purizaca N, López R, Lajo Y, Peña E, Apolaya M, Díaz C. Factores asociados con el nivel de conocimientos y la actitud hacia la investigación en estudiantes de medicina en Perú, 2011. Revista Panamericana de la Salud Publica. 2013;33(3).

15. Díaz C, Manrique L, Galán E, Apolaya M. Conocimientos, actitudes y prácticas en investigación de los estudiantes de pregrado de facultades de medicina del Perú. Revista Acta Med Per. 2008; 25(1):325-29.

16. Jurado S. Actitudes hacia la investigación en estudiantes de enfermería de la UNMSM, 2016 [Tesis de licenciatura]. Lima: Universidad Nacional Mayor de San Marcos; 2017.

17. Arteaga M, Alvarado G. Factores que influyen en la elaboración de una tesis para obtener un título en estudiantes de las universidades de Trujillo.2015;26(2). 